\title{
العلاقات بين الدول الإسلامية
}

المؤلف: أ. د. محمد السيد سليم.

الناشر: عمادة شؤون المكتبات، جامعة الملك سعود (الرياض)، 1991.

عبده عمر شورى

تقديم

يحتوي الكتاب الذي بين أيدينا على ستة فصول، ويشتمل كل فصل منها على مباحث ومطالب. وفضلاً عن ذلك يتضمن الكتاب ثمانية ملاحق. وليست هناك خاتمة أو خلاصة للكتاب. ففي الفصل الأول يتناول المؤلف تعريف الدول الإسلامية، وفي الثاني خصائص تكوينها، وفي الثالث التطور التاريخي، وفي الرابع النظرية السياسية للعلاقة بين الدول الإسلامية، وفي الخامس العلاقات المعاصرة، وفي السادس الأطر التنظيمية للعلاقات.

ومن الملاحق: شبكة العلاقات الدبلوماسية بين الدول الإسلامية سنة 1970م، وميثاق منظمة المؤتمر الإسلامي، واتفاقية إنشاء البنك الإسلامي للتنمية.

وفصول الكتاب في أصلها بجموعة من المحاضرات التي ألقاها لمؤلف على طلاب كلية العلوم الإدارية بجامعة الملك سعود في مقرر العلاقات بين الدول الإسلامية وقدر جرى تحريرها وإعدادها للنشر العام.

وفيما يخص منهج الكتاب ليس فيها عيب، إلاّ أن بعض الفصول تقع في غير موضعها في الترتيب، فمثلاً الفصل الثالث: "التطور التاريخي للعلاقات بين الدول الإسلامية"، لا يمكن أن يأتي بعد الثاني لأنه في التطور التاريخي للظاهرة. والغريب في الأمر أنه ليست هناك خاتمة للكتاب ولا أدري قصد المؤلف من ذلك. كما أن الملاحق احتلت ما لا يقل عن تسع وخمسين صفحة من حجم الكتاب مما يسبب خلاًً واضحاً في بنيته. 
ومن الأشياء التي تعطي هذا الكتاب أهمية علمية في تقديري:

1- الموضوع الرئيسي (main theme) للكتاب يكاد يكون من الموضوعات التي تنعدم فيها الدراسات العلمية الجادة وخاصة باللغة العربية.

2- طريقة تحليل المؤلف للظاهرة وتصنيفه للأفكار أمر متميز وفيه قدر كبير من الأصالة، لا سيما إذا قارنا الكتاب بعدد من المؤلفات التي صدرت باللغة العربية في هذا الموضوع. 3- خبرة المؤلف في التدريس في هذا الموضوع وفرت له مادة غنية وسعة نظر فيها.

والكتاب المتميز كذلك من حيث الطريقة التي بنى عليها المؤلف تحليلاته والأصول التي انطلق منها لفهم الظاهرة المدروسة: العلاقات بين الدول الإسلامية. وهو إسهام جديد في الميدان، ودعوة الباحثين المسلمين إلى الاهتمام بمثل هذه الدراسات، لأن العلاقات السياسية في العالم الإسلامي واقع لا يمكن تجاهله، وهي ظاهرة تحتاج إلى فهم ودراسة وتحليل.

\section{الفصل الأول: تعريف الدولة الإسلامية}

والدولة الإسلامية في رأي المؤلف: "كيان سياسي قانوني ينصرف إلى مجموعة الأفراد الذين يعيشون في إقليم

$$
\text { واحد موحد وتحكمه سلطة سياسية ذات سيادة". (ص 1) }
$$

ويدعو المؤلف بالباحثين إلى أن يفرقوا بين مفهوم "العالم الإسلامي" ومفهوم "الدولة الإسلامية" لكوغما وحدتين مختلفتين. "فالدول الإسلامية عبارة عن كيانات سياسية قانونية قد تضم بعض الشعوب والأقليات غير المسلمة. أما مفهوم العالم الإسلامي فإنه ينصرف إلى الوجود الإسلامي في كل مناطق العالم بصرف النظر عما إذاكان

$$
\text { هذا الوجود يتم في إطار الدول الإسلامية أو غير الإسلامية". }
$$

من هنا يأتي الإشكال حول تعريف المؤلف للدولة الإسلامية. فالقارئ يلاحظ -بدون صعوبة- أن المعيار الذي يستخدمه هو عبارة عن إضفاء الشرعية على الحكومات القائمة في ديار المسلمين وتبرئتها من عمة عدم 
المشروعية التي يوجهها إليها العديد من الفقهاء والمفكرين. كذلك يدرك القارئ أن المؤلف اختلطت عليه المفاهيم حول "الدولة المسلمة" و"الدولة الإسلامية". ففي العالم الإسلامي دول مسلمة وليس هناك دولة إسلامية، وليس هناك قانون إسلامي قُدّم من قبل الفقهاء والعلماء، يمكن تطبيقه على العالم الإسلامي، صالح للشعوب المختلفة. وهذه على أية حال مسألة جرى فيها اختلاف كبير بين المفكرين والفقهاء المعاصرين.

والجدير بالذكر أن صميم التعريف الذي قدمه المؤلف فيه نظر، ذلك لأن هذا التعريف عام، وشامل، ولا يختلف عن بعض التعريفات التي قال بها علماء السياسة في الغرب مثل بودين (1596-1530) Jean bodin وهو أول من عرّف الدولة (state)، وروسو (1778-J.J. Rousseau ولوك John lock (1712) (1704-1632)، أو التعريفات التي قدمها مفكرو المسلمين مثل ابن خلدون، (1406-1332). هذا وفي محاولة للخروج من هذا الإشكال لجأ الكاتب إلى سَوْق العديد من التبريرات منها أنه لا يمكن تحديد هوية الدولة الإسلامية، وأنه يجب التمييز بين التعريف النظري للهوية والتطبيق العملي لتلك الهوية في تنظيم شؤون الحكم والمجتمع في إطار الدولة. وكثيراً ما يتجنب الكاتب التعريفات التي قُدمت من قبل المفكرين الإسلاميين والفقهاء قديماً وحديثاً، ويزيد الإشكال السابق حدةً عندما يؤكد المؤلف في هذا الفصل أن: "الدولة الإسلامية هي الدولة المسلمة ولا فرق بين الاثنتين"، وأن نسبة المسلمين في الدولة لا تؤثر في إسلامية الدولة والعكس صحيح، "فالدولة الإسلامية هي التي تعرف إزاء العالم الخارجي أهما إسلامية، أي أها تلك الدولة التي تعرف النخبة الحماكمة فيها هوية للدولة على أها دولة إسلامية حتى إذا لم تكن قضية تطبيق الشريعة الإسلامية مثارة فيها كإحدى أولويات السلطة السياسية". (ص 8) (n)

\section{الفصل الثاني: خصائص تكوين الدولة الإسلامية}

وقد عرض المؤلف آراء مهمة خاصة بالجغرافيا السياسية (geopolitics) للدول الإسلامية، وبخصائصها الاجتماعية والاقتصادية، والمؤثرات الداخلية والخارجية في العلاقات بين تلك الدول. وناقش كذلك موقع الدول الإسلامية جغرافياً حيث إها تشمل مساحة واسعة تمتد من المحيط الأطلسي غرباً (المغرب وموريتانيا والسنغال) إلى المحيط الهادي شرقاً (إندونيسيا وماليزيا وبروناي)، ومن بحر قزوين شمالاً (إيران وتركيا) إلى بحيرة فيكتوريا جنوباً (أوغندا). وهي مساحة لا تقل عن 36.618 مليون كيلومتر مربع، وتمثل 30.04 ٪ من مساحة اليابسة في العالم. 
ويُعتبر السودان أكبر الدول الإسلامية مساحةً (3.5.6 مليون كيلومتر مربع)، بينما تُعتبر جزر المالديف أصغرها مساحةً (300 كيلو متر مربع).

والحقيقة أن المؤلف قد قدم هنا تحليلاً جغرافياً دقيقاً معتمداً فيه على أعمال عدد من الباحثين الجغرافيين من المسلمين المعروفين منهم: جمال حمدان في كتابه: العالم الإسلامي المعاصر، وصلاح الدين علي الشامي وزين الدين عبد المقصود في كتابمما: جغرافية العالم الإسلامي، وجودة حسن جودة في مؤلَّف: جغرافية الدول الإسلامية، وعبد الرزاق الجوهري مؤلف كتاب: جغرافية السعودية الإسلامية، وغيرهم.

وينبغي أن نلاحظ هنا أن الموقع الجغرافي الاستراتيجي لا قيمة له في حد ذاته إذا لم يُستفد منه أمنياً وسياسياً واقتصادياً. فبلدُ كالصومال مثلاً ذو موقع من أهم المواقع بالنسبة إلى العالم الإسلامي وغير الإسلامي، ولكن أهله لم يستطيعوا توظيف هذا الموقع الممتاز لخدمة مصالحهم. لكن ينبغي أن نلاحظ مع ذلك أن تطور تقنيات الصواريخ العابرة للقارات وتقنيات الطيران قد أدى إلى تضاؤل أهمية الموقع الجغرافي، فقد يكون لدولة معينة موقع استراتيجي مهم من النواحي السياسية والعسكرية والاقتصادية ولكنها تعجز تماماً عن حماية نفسها من الأعداء، خصوصاً إذاكان الأعداء من القوى الكبرى التي بإمكاها أن تنتقل بأسلحتها وعتادها إلى أبعد الأماكن وفي أقصر وقت ممكن، كما حدث إبّان الصراعات الأخيرة بين الولايات المتحدة الأمريكية والعراق أيام حرب الخليج.

إن العالم الإسلامي -والمؤلف يرفض استخدام عبارة العالم الإسلامي في هذا الكتاب- يعاني حسب اعتقاد الكاتب آثار مشكلتين جغرافيتين: أولاهما الاتساع الجغرافي، ويقصد به أن الدول الإسلامية تمتد امتداداً أفقياً واسعاً ولا تزال تعاني ضعفاً واضحاً في وسائل الاتصال بكل أنواعه وخاصة الاتصال الإلكتروني. فلم تصل شبكة الاتصالات الدولية (internet) إلى اليوم إلى كثير من الدول الإسلامية، وكذا آلة الحاسوب. بل إن الطاقة الكهربائية التي هي الأساس لاستخدام هذه التقنية الحديثة تعاني تخلفاً ملحوظاً في هذه الدول.

والمشكلة الثانية: هي أن الدول الإسلامية بما مساحات شاسعة من الأراضي الصحراوية، وذلك على حساب المساحات القابلة للزراعة التي لا تزيد عن 8.9٪. 
أما عن الخصائص الاجتماعية والاقتصادية للدولة الإسلامية، فقد تناول المؤلف إحصائيات السكان بالدول الإسلامية بما فيها فلسطين وإندونيسيا وجزر المالديف، فأوضح أنّ إندونيسيا تشكل 19.8 ٪ من سكان الدول الإسلامية. ثم حاول أن يحدد الوضع الاقتصادي العام للدول الإسلامية من خلال بعدين رئيسين هما: التخلف والتنمية الاقتصادية، والتبعية والاستقلال الاقتصادي، إذ أن هذين العاملين يؤثران تأثيراً عميقاً في العلاقات بين الدول الإسلامية بأشكال مختلفة، ومن ثّ انتقل المؤلف لمناقشة العلاقات بين الدول الإسلامية.

وفي مقدمة هذا النقاش تساءل المؤلف عما إذا كان هناك منظور تاريخي للعلاقات بين الدول الإسلامية. ولإجابة عن هذا التساؤل تناول المؤلف التطورات التاريخية والسياسية التي مرت بالعالم الإسلامي منذ قيام الخلافة العباسية عام 750م إلى سقوط الدولة العثمانية عام1924 م، فميّز بين مراحل خمس أساسية ومهمة في تطور علاقات الدول المسلمة مع الخلافة العامة وهي: 1- المرحلة التي شهدت نشأة الدولة العباسية وسقوطها (من القرن الثامن إلى الثالث عشر). 2- المرحلة التي اهارت فيها الدولة العباسية، وقامت الدولة العثمانية (من القرن الثالث عشر إلى القرن السادس عشر). حيث ظهرت بصورة جلية سيطرة العثمانيين.

3- التكالب الاستعماري على العالم الإسلامي (من القرن السادس عشر إلى التاسع عشر). 4- ظهور حركة الجامعة الإسلامية (من القرن التاسع عشر إلى غاية الحرب العالمية الأولى). 5- ظهور حركة الجامعة الإسلامية القطرية (Muslim Nation-States) (ص 61). لقد أشار الكاتب إلى أن الباحثين الغربيين قد حللوا التطورات السياسية والتاريخية مثل "صلح ويستفاليا" عام 1648م. ولكنَّهم لم يلتفتوا إلى أوضاع الدولة المغولية في الهند والدولة الفارسية وأحوالهما السياسية في ذلك العهد. وحين عرضوا تطورات مؤتمر فيينا عام 1815م كُفّت أبصارهم، ولم يُعطوا العلاقات بين الدولة العثمانية والدولة الفارسية القاجارية في المرحلة ذاتا طرفة عين. 
ويواصل المؤلف نقاشه حول تحليل العلاقات بين الدول الإسلامية التي قامت إثر ضعف الدولة العباسية وسقوطها عام 1858م حيث أشار إلى وجود ست دول إسلامية نشأت بعد اهيار الدولة العباسية هي:

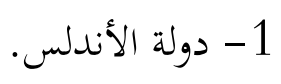

2- 2 دولة الموحدين في شمال إفريقيا.

3- 3 دولة المماليك في مصر والشام.

4- 2- دولة السلاجقة الروم في الأناضول.

5- الدولة المغولية التركية في آسيا الوسطى وشمال الهند.

$$
\text { 6- إمبرطورية مالي في غرب إفريقيا. }
$$

وفي هذا الصدد ذهب المؤلف إلى أن العلاقات بين هذه الدول لم تكن قائمة على الانسجام والتكامل، بل كانت تنكر بعضها بعضاً، على الرغم من أنه قد حاول من خلال نقاشه أن يؤكد وجود بعض الإيجابيات في هذه العلاقات أكثر من السلبيات.

ففي عام 1380م استولى المغول على أفغانستان وبلاد فارس والعراق وجورجيا وأرمينيا وكردستان. وبعد ثلاثة عشر عاماً من ذلك التاريخ دخل القائد المغولي تيمورلنك في صراع مسلح مع دولة المماليك والدولة العثمانية حتى استولى على الشام بأكملها. وسقط السلطان بايزيد الأول أسيراً في يد تيمورلنك في معركة أنقرة عام 1403م. وملا قويت الدولة العثمانية عسكرياً بقيادة محمد الفاتح وتأسست الدولة الصفوية الشيعية حصل الصدام بين المماليك والعثمانيين، وذلك في بداية القرن السادس عشر حيث وجد نظام توازن القوى (Balance of Power). ويبقى السؤال أين العلاقات الإيجابية بين الدول الإسلامية التي يتحدث عنها المؤلف؟ ومن القضايا التي ناقشها المؤلف في كتابه قضية "الجامعة الإسلامية (Pan-Islamism)، فما هي الجامعة

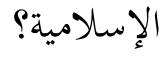


برزت الفكرة مع الشيخ جمال الدين الأفغاني، ثم تبناها عبد الحميد الثاني من ناحية عملية حتى يوطد سياسته ويعزز ملكه. تم ماتت الفكرة بين يديه، وظهرت بعد موقها أربعة تيارات في العالم الإسلامي. تيار وطني قطري يمثله أحمد لطفي السيد في مصر، وتيار عروبي ينادي بالقومية العربية وعلى رأسه نجيب عازوري في لبنان، وتيار عروبي إسلامي قاده عبد الرحمن الكواكبي، وأخيراً تيار السلطان عبد الحميد الثاني الذي نادى "بالجامعة الإسلامية" بعد أن حورها عن طريقها الأصلي ثم استخدمها لتحقيق مصالحه السياسية. وبقرار من الحكومة التركية بقيادة مصطفى كمال أتاتورك انتهت الخلافة، وبدأت الدعوة لإعادة الخلافة الإسلامية من جديد.

\section{الفصل الرابع: النظرية السياسية للعلاقات بين الدول الإسلامية}

حاول المؤلف تقديم صورة متكاملة عن ظاهرة التعدد السياسي في العالم الإسلامي من خلال عرض آراء المفكرين المسلمين في هذه الظاهرة وبيان ما هو واقعي (realistic) منها ما هو مثالي (idealistic). وفي رأيي أن المبحث الأول هو أهم مباحث هذا الكتاب، فهو يمثل الأفكار الأساسية للكتاب، ولا أدري لماذا لم يضعه المؤلف في آخر الكتاب حتى يكون بمنزلة خاتمة مركزة للموضوع الذي دار عليه الكتاب. هذا وقد بدا المؤلف بوضع مقدمات عامة عن وحدة الأمة الإسلامية، على أساس أن الأمة جماعة من الناس تربطها عقيدة واحدة، وشريعة شاملة، إلا أنه يعتقد أن ذلك لا يعني "بالضرورة" وحدة قيادة الأمة. فهو يرى أن الآيات القرآنية والأحاديث النبوية التي وردت في هذا إنما تتحدث عن الروابط الروحية والعقدية بين المسلمين (ص 118). والذي يفهم من كلام المؤلف هذا أن وحدة الخنافة أو الرئاسة العامة ليست ركناً أساسياً في التراث السياسي الإسلامي، وأن المؤسسات السياسية المسلمة المعاصرة جائزة في الشرع والعقل لتمثل الأمة في صورها الراهنة. والفقهاء في المسألة على مذهبين: مذهب يؤيد وجود أكثر من خليفة في البلاد الإسلامية في وقت واحد وقد ذهب إلى ذلك البغدادي والجويني، ومذهب يرى اشتراط وحدة الرئاسة في البلاد الإسلامية وقد قال بذلك الماوردي. وحجة هؤلاء من السنة قول الرسول صلى الله عليه وسلم: "من أتاكم وأمركم جميع على رجل واحد يريد أن يشق عصاكم، أو يفرق جماعتكم، فاقتلوه".

والغريب في الأمر أن المؤلف قد فسر هذه الأحاديث على أها تتعلق بشرعية الثورة على الخليفة فقط. وقد

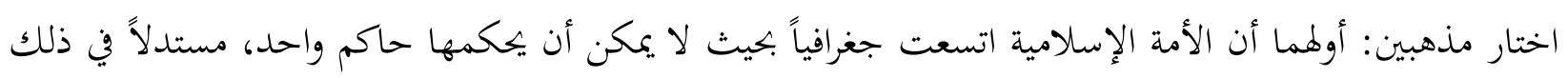
برأي الدكتور عبد الحميد أبو سليمان (مدير الجامعة الإسلامية العالمية بماليزيا) في رسالته للدكتوراه عن النظرية 
الإسلامية للعلاقات الدولية، حيث قال: "إن رسول الله صلى الله عليه وسلم حينما نادى بالوحدة من بعده إنما كان يشير إلى ضرورة المحافظة على النظام السياسي الذي أقامه في المدينة وليس إلى إقامة سلطة سياسية واحدة تضم جميع

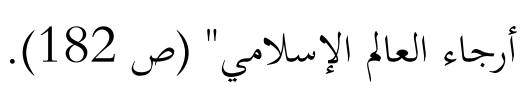

وقد استند إلى أقوال مفكرين مسلمين معاصرين آخرين مثل الشيخ محمد أبي زهرة حيث قال: "إن الوحدة الإسلامية ليست دولة واحدة".

وفي رأي المؤلف أن إنكار أبي بكر على ابن المنذر عندما طالب بأن يكون من الأنصار أميرٌ ومن المهاجرين

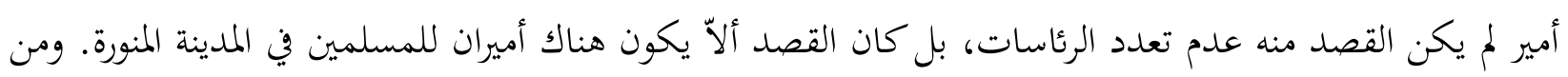
المبادئ التي يؤكدها الإسلام في نظر المؤلف الأخوّة الإسلامية، ونبذ العصبية، والنصح المتبادل، والجماعة الإسلامية المتكافلة، وعدم الاعتداء بين الدول الإسلامية، والتضامن الجماعي، وأسس فض المنازعات بين الدول الإسلامية. وبذلك ليس لمبدأ وحدة الرئاسة وجودٌ بين المبادئ التي أقرها الإسلام وحث عليها.

ومن هنا يمكن القول -بناءً على تحليلات المؤلف- إن كل مفكر إسلامي معاصر ينادي في العصر الحديث بوحدة القيادة الرئاسية في العالم الإسلامي إنما هو مثالي وليس واقعياً. فجمال الدين الأفغاني رجلٌ مثالي، والشيخ رشيد رضا رجلٌ مثالي، والشيخ حسن البنا رجلٌٌ مثاليٌ أيضاً. في حين أنَّ الشيخ محمد عبده، والشيخ عبد الرحمن

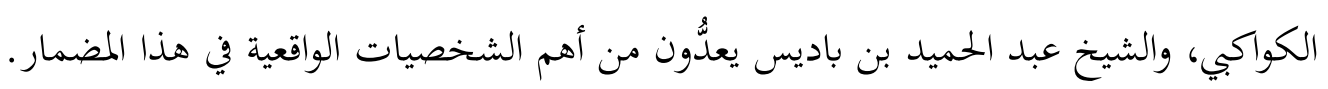

وللعلاقات الاقتصادية والسياسية والدبلوماسية بين الدول الإسلامية المعاصرة أهمية خاصة في نظر المؤلف. حيث يرسم في توضيحه بعض الرسومات البيانية التي توضح المسائل الاقتصادية والجغرافية والسياسية في هذا السياق. فتاريخ العلاقات بين الدول الإسلامية يدل دلالة واضحة على أها أحيت فكرة القومية وأماتت فكرة التضامن الإسلامي. إلاّ أن فكرة التضامن غضت من جديد في بداية الستينيات لأها لم تمت موتاً غائياً. وكادت أن تتحدى

The Islamic Theory of International Relations: New : أعدت هذه الرسالة باللغة الإنجليزية تحت عنوان Directions of Islamic Methodology and Thought

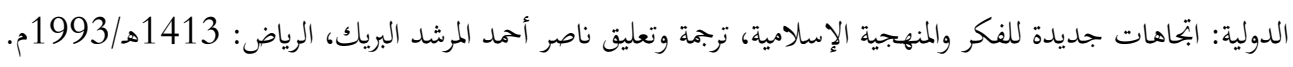


الأفكار السياسية الأخرى في العالم الإسلامي مثل القومية والعلمانية والأيديولوجيات والنظم السياسية التي نتجت من تلفيق المذاهب الغربية وتقليدها مثل الشيوعية والديمقراطية.

وفي غاية هذه الرحلة ناقش المؤلف كيفية إنشاء منظمة المؤتمر الإسلامي والبنك الإسلامي للتنمية، وأثرها وأهداف كل منها. وما زلتُ أتأمل وأتساءل حول مدى إمكانية أن تواصل هذه المنظمات أعمالها حتى تؤدي واجبها المنوط بها؟ وهل تسير منظمة المؤتمر الإسلامي على النهج القويم الذي يؤدي إلى توجيه الأمة توجيهاً صحيحاً وإبراز أثر سياسي يتجاوز التكوين القومي والمصالح الوطنية؟ وإلى متى نتظر حتى تنمو شوكتها وتقوى على اتخاذ القرارات الموحدة والحاسمة حول الأزمات الراهنة؟ وهذه كلها قضايا مهمة لا أرى سبباً يجعل المؤلف لا يلتفت إليها في كتابه. 